\title{
Analytical Formulae for Calculating SRM Modal Frequencies for Reduced Vibration and Acoustic Noise Design
}

\author{
W. Cai \\ Delco Remy America, Inc. \\ 2902 Enterprise Drive, \\ Anderson, IN 46013, \\ email: caiw@delcoremy.com
}

\author{
P.Pillay \\ Department of ECE \\ Clarkson University \\ Potsdam, NY 13699 \\ email: pillayp@clarkson.edu
}

\author{
A.Omekanda \\ Delphi Research Labs \\ 51786 Shelby Parkway \\ Shelby Township, MI 48316 \\ avoki.omekanda@delphiauto.
}

\begin{abstract}
Electronic techniques for controlling acoustic noise and vibration depend on an accurate knowledge of the stator resonant frequency. Analytical models are developed, which allows the calculation of the first several modal frequencies. The impact of the stator stack length on the accuracy of the formulae is also examined. Experimental validation is included.
\end{abstract}

\section{INTRODUCTION}

One of the disadvantages of the Switched Reluctance Motor (SRM) is its vibration and corresponding acoustic noise. Many of the methods to reduce acoustic noise of the SRM, like Random Pulse Width Modulation (RPWM) or active noise control using two or three stage commutation, assume that the resonant frequency of the stator is known or measurable. It is essential to be able to predict the resonant frequencies and the response characteristics of the stator in order to design a quiet SRM or to avoid operating the motor near the resonant frequency during drive operation. The mode shape provides valuable information as to the excitation pattern to produce a particular resonance.

The resonant frequencies can be obtained by analytical calculation, numerical computation (finite element method etc.) and/or experimental techniques. The experimental method can be performed only after a prototype has been built. Therefore, deriving analytical formulae and building numerical computation models, which have been validated experimentally, are essential for motor designers. Because of the complexity of numerical computation, analytical methods are more popular in the early stages of motor design. The tradeoffs between different design parameters are easier to analyze with analytical models. There are advantages in combining the analytical method with numerical computation, i.e., to use the numerical methods to correct the deviation from analytical predictions due to simplifications necessary in the analytical model.

This paper firstly derives a formula to predict the second order resonant frequency of stator laminations. This is usually the resonant frequency of most interest. The

formula can also be used in cases where smooth frame structures are used to support the stator lamination stack. A more general formula is also developed to calculate the integer order resonant frequencies of the first few mode shapes. The analytical formulae are compared with a more accurate calculation using the finite element method and experimental results. The applicable range of the formulae is discussed, which act as a guide to designers in the use of these formulae.

\section{DERIVATION OF MODAL FREQUENCY FORMULAE}

\section{Formula for the frequency of the $2^{\text {nd }}$ order mode}

The method of computing approximate natural frequencies of a vibration system, using an energy approach, has become known as 'Rayleigh's method". The resonant frequency of the fundamental mode (the $2^{\text {nd }}$ order mode) is obtained by equating the peak kinetic energy $T_{\max }$ with the potential energy of the maximum deformation $U_{\max }$ when the stator lamination stack is modeled as a slender cylindrical shell. The derived formula for the $2^{\text {nd }}$ order modal frequency is given as follows

$$
f_{2}=\frac{\omega_{2}}{2 \pi}=\frac{k_{c}}{2 \pi} \frac{h_{y}}{R_{y(a v)}^{2}} \sqrt{\frac{E}{\rho_{F e}}}
$$

where $\omega_{2}$ is the angular frequency; $h_{y}$ is the thickness of stator yoke; $R_{y(a v)}$ is the average radius of yoke; E is Young's modulus of the stator lamination material; $\rho_{F e}$ is the mass density of the lamination materials; and the coefficient $k_{c}$ can be expressed as follows:

$$
k_{c}=\left[\frac{\frac{1}{2} k_{1}-\frac{4}{\pi^{2}} k_{2}^{2}}{3\left(1-v^{2}\right)\left(\frac{\pi}{4} k_{1}+\frac{1}{2} k_{1}-k_{2}\right)^{2}}\right]^{1 / 2}
$$

where $v$ is the Poisson's ratio and the coefficients $k_{1}, k_{2}$ can be expressed by Poisson's ratio and Hoop-stress deformation factors $\alpha$ :

$$
\begin{aligned}
& k_{1}=1+\frac{1}{5}(7+12 v) \alpha \\
& k_{2}=1-\alpha
\end{aligned}
$$




$$
\alpha=\left\{\begin{array}{cc}
1-\frac{h_{y}}{R_{y(a v)} \ln \left[\left(\frac{R_{y(a v)}}{h_{y} / 2}+1\right) /\left(\frac{R_{y(a v)}}{h_{y} / 2}-1\right)\right]}, 0.6<\frac{R_{y(a v)}}{h_{y}} \leq 8 \\
\frac{1}{12\left(1-v^{2}\right)}\left(\frac{h_{y}}{R_{y(a v)}}\right)^{2}, & \frac{R_{y(a v)}}{h_{y}}>8
\end{array}\right\}
$$

$\alpha$ is not equal to zero for the common dimensions of SRM stator. When $R_{y(a v)} \rightarrow \infty$ (a straight beam) for given yoke thickness $h_{y}, \quad \propto 0$ and $k_{1} \approx k_{2} \approx l$ which was used in [4]. An error in the deformation calculation can occur if $k_{1} \approx k_{2} \approx 1$ is used without checking the ratio $\left.R_{y(a v}\right) h_{y}$.

Equation (1) is also called the "thick pipe model". When the average radius of the stator yoke is much larger than the yoke thickness, i.e., $R_{y(a v)}>>h_{y}$, the coefficients $k_{1} \approx k_{2} \approx 1$. Furthermore taking Poisson's ratio $v=0.3$, the coefficient $k_{c}=0.6526$, which makes equation (1) identical to the formula in reference [4].

If $\left(1-v^{2}\right)$ in Eq.(2) \& Hook deformation coefficient expression is replaced by 1 , the corresponding model is called the "thin ring model".

\section{FREQUENCY ESTIMATION OF MULTI-ORDER MODES}

Equation (1) can be used to approximately estimate the frequency of the $2^{\text {nd }}$ order vibration mode of SRM stator. The method for accurate determination of the resonant frequency of a complex stator structure in an induction motor has been described [8]. An alternative formula for modal frequencies of the $\boldsymbol{r}$ th order mode is derived [1] and given below

$$
f_{r}=\frac{1}{2 \pi} \sqrt{\frac{k_{r}}{m}}
$$

Where $m$ is the equivalent mass per square meter $\left(\mathrm{kg} / \mathrm{m}^{2}\right)$ on the cylindrical surface at average yoke radius, which can be expressed by the following formula:

$$
m=\frac{M_{y}}{2 \pi R_{y(a v)} L_{y(e f f)}}
$$

and the equivalent spring stiffness coefficient per square meter $k_{r}\left(\mathrm{~N} / \mathrm{m}^{3}\right)$ in Eq.(3) on the cylindrical surface at average yoke radius can be written as $[1,9,10]$

$$
k_{r}= \begin{cases}\frac{E h_{y}}{R_{y(a v)}^{2}} & \text { for } \mathrm{r}=0 \\ k_{D}\left(k_{D}=0 \text { withoutshockabsorber }\right) & \text { for } \mathrm{r}=1 \quad(5) \\ \frac{r^{2}\left(r^{2}-1\right)^{2}}{\left(1+r^{2}\right)} \frac{E h_{y}^{2}}{12 R_{y(a v)}^{4}} & \text { for } \mathrm{r} \geq 2\end{cases}
$$

It should be noticed that the formulas (3) (5) can be applied to predict only the natural frequencies of the first several low order vibration modes. They may lead to unacceptable errors if the formulas are used to calculate the mode greater than the $5^{\text {th }}$ order. This is shown in the comparison with the numerical method and the measured values.

\section{PRACTICAL CALCULATION AND RESULT COMPARISON}

In order to verify the validity of the above formulas, extensive numerical calculations and an experimental investigation were carried out on a $4 \mathrm{~kW}, 8 / 6$ pole, 4 phase switched reluctance motor. The resonant frequency results of the $2^{\text {nd }}$ order mode from the analytical models are compared with the corresponding results from the finite element method, as shown in Table 1. The finite element result $(817.3 \mathrm{~Hz})$ is based on the 3D free vibration model. The errors of the analytical models are within the allowable range of engineering error.

Effect of Lamination Length: To explore the effects of stator lamination length on the resonant frequency of the $2^{\text {nd }}$ order in-plane vibration, the $2^{\text {nd }}$ order frequencies are calculated by FEM through the lamination length range of $89.8 \sim 190 \mathrm{~mm}$ while the cross section dimensions of the stator laminations are kept unchanged. The finite element results are given in Fig.1.

The $2^{\text {nd }}$ order resonant frequencies in Fig. 2 show only a slight variation $(0.54 \%)$ with change of stator lamination length when the ratio between length/radius varies from 1 to 2. Furthermore, the resulting errors of the analytical

\section{TABLE 1. COMPARISON OF FREQUENCY RESULTS BETWEEN MODELS}

\begin{tabular}{|l|c|c|c|}
\hline Models & Thick Pipe & Thin Ring & Multi-order \\
\hline Frequency $(\mathrm{Hz})$ & 858.3 & 818.7 & 790.2 \\
\hline Error $*(\%)$ & 5.02 & 0.002 & -3.32 \\
\hline
\end{tabular}

<*> The base value is FE result $817.3 \mathrm{~Hz}$

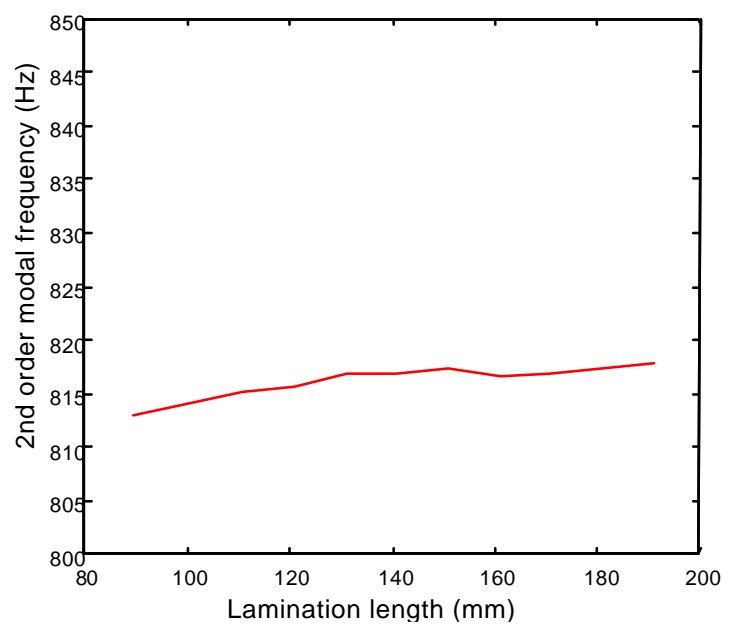

Fig. 1 The $2^{\text {nd }}$ order modal frequency vs. lamination length by FE 


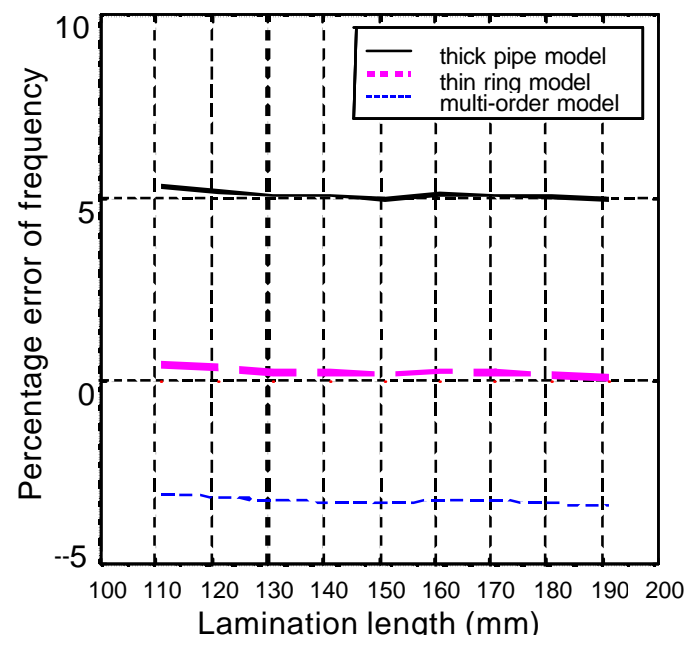

Fig.2 Percentage error of frequency vs. lamination length

formulas with lamination length changes, compared with that by numerical calculations, are demonstrated in Fig.2. All the 2D analytical formulas for analyzing in-plane flexural modes in the paper are accurate enough to satisfy industrial applications.

Effect of Stator Yoke Radius/Thickness: To search for the applicable range of the analytical formulas presented in the paper, the $2^{\text {nd }}$ order resonant frequencies of the stator lamination are calculated through a range of yoke radius of $85.8 \sim 99.8 \mathrm{~mm}$. In the calculation, the length of the laminations is kept at the actual length of the stator stack $(151 \mathrm{~mm})$ of the $4 \mathrm{~kW}$ SRM. The curves of the relationship between the $2^{\text {nd }}$ mode frequency and yoke outer radius are shown in Fig.3. Compared with the frequency values from the finite element method, the errors of the three analytical

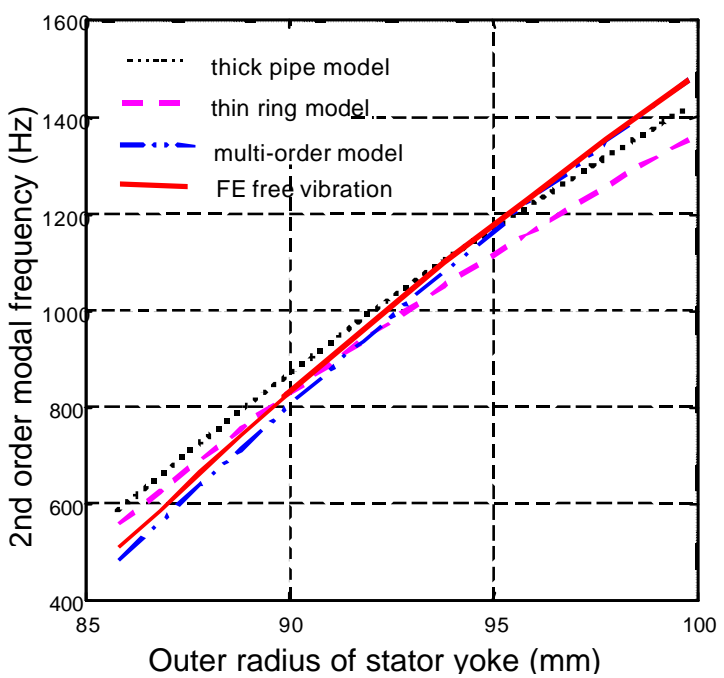

Fig. $32^{\text {nd }}$ order modal frequency vs. yoke radius when the lamination length is kept unchanged

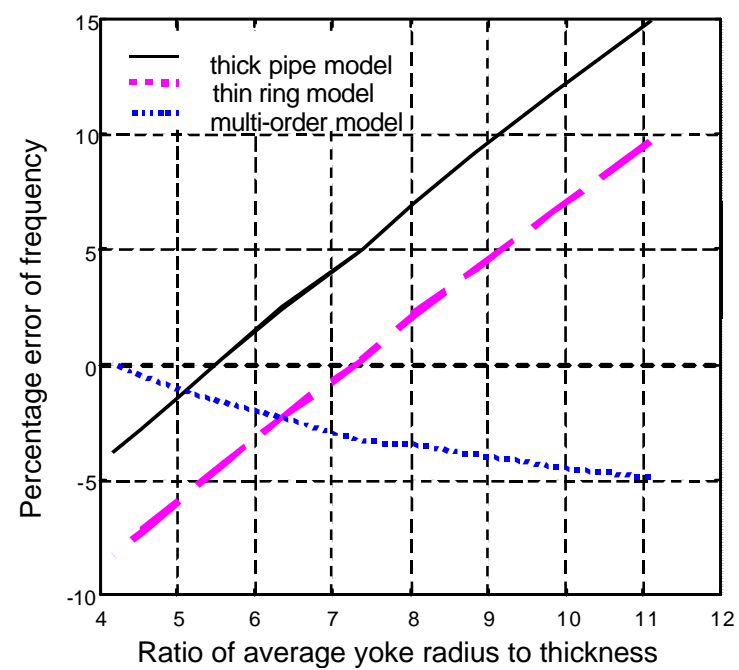

Fig.4 Percentage error of frequency vs. ratio of yoke radius/thickness when lamination length $=$ const

models are drawn in Fig.4. In this figure, the ratio of average yoke radius to thickness is taken as the abscissa in order to generalize the applicable range of the analytical formulas. The ratio from 4.16 to 11.1 corresponds to the outer radius range of stator laminations from 99.8 to $85.8 \mathrm{~mm}$ in the real $4 \mathrm{~kW}$ SRM when its yoke inner radius fixed at $78.4 \mathrm{~mm}$.

From Fig.4, the frequency errors are within 5\% when the radius/thickness of the stator yoke falls in the range of 4 7.3 in the thick pipe model, 5.4 9.2 in the thin ring model, $4 \sim 12$ in the multi order model. Within these ratio ranges, the corresponding formulas meet the needs of engineering requirements for estimation of the $2^{\text {dd }}$ resonant frequency. The applicable range for frequency estimation by the multi order model seems much wider.

Low Order Frequency Estimation: The formulas of the multi order model can theoretically be applied to predict the frequencies of integer order vibration modes, but the real calculation shows only limited order frequencies may be estimated by the formulas (3) (5). The frequency errors produced by the multi order model are shown in Fig.5, compared with the values with finite element results. The mode shapes of the vibrations include the $0^{\text {th }}, 2^{\text {nd }}, 3^{\text {rd }}$ and $4^{\text {th }}$ order, whose deformations are shown as in Fig.6. The 1st order mode reflecting free vibration of the stator yoke can be estimated by reference [10] if the feet of the motor are fixed on an infinite base, which is similar to the rotating vibration under mass unbalance of rotor. From Fig.5, the percentage errors of the resonant frequencies from formulas (3) (5), can be restricted to within $5 \%$ if the radius/thickness of the stator yoke falls into the following ranges: $4 \sim 8.8$ range for the $0^{\text {th }}$ order, $4.2 \sim 11$ for $2^{\text {nd }}$ order, more than 8.5 for the $3^{\text {td }}$ order, and $4.4 \sim 7.5$ for the $4^{\text {h }}$ order. Obviously these formulas are accurate enough to predict the frequencies of the $0^{\text {th }}, 2^{\text {nd }}$ and some $4^{\text {th }}$ order vibration modes. But it should be noticed that 


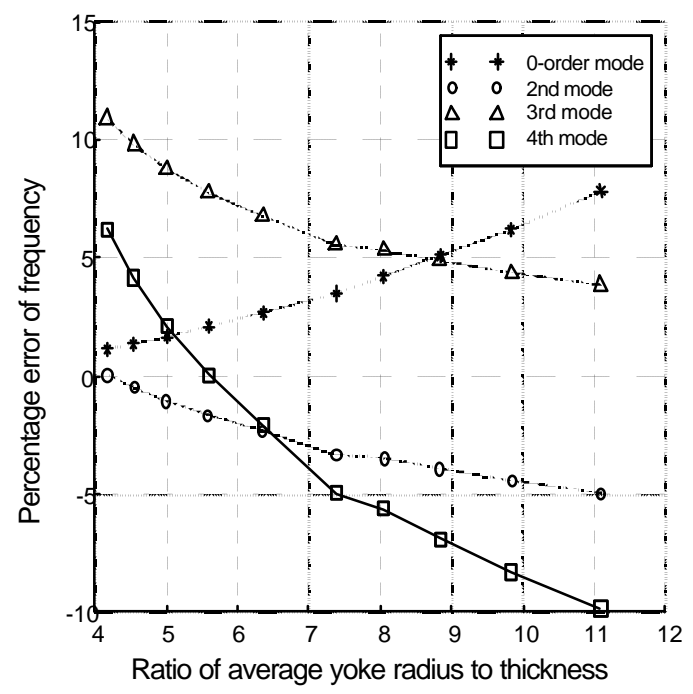

Fig.5 Percentage error of frequency vs. yoke radius/thickness under multi order model (order $0,2,3,4$ )

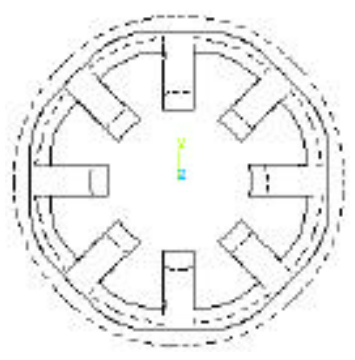

(a) $0^{\text {th }}$ order mode

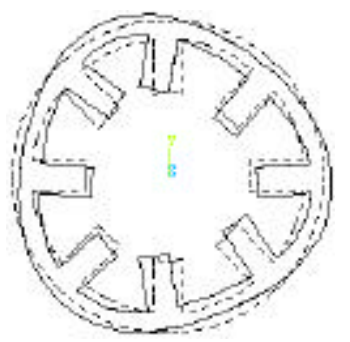

(c) $3^{\text {xd }}$ order mode

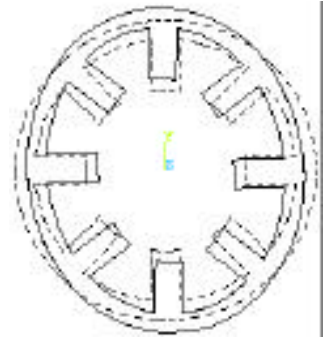

(b) $2^{\text {nd }}$ order mode

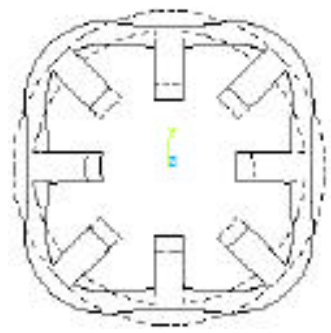

(d) $4^{\text {th }}$ order mode
Fig.6 Deformation of 4 order mode shapes

the multi order model brings unacceptable errors if it is used to calculate the frequency beyond the $5^{\text {th }}$ order vibration mode.

\section{COMPARISON WITH MEASUREMENTS}

To verify the validity of the formulas, the calculated values of the $2^{\text {nd }}$ order modal frequency is compared with the measurement results of a $4 \mathrm{~kW}$ SRM. The contact between laminations and case is assumed to be perfect due to the encased structure. The frequency measurements are performed with an accelerometer behind the stator pole while the phase windings on these two poles are conducting pulse
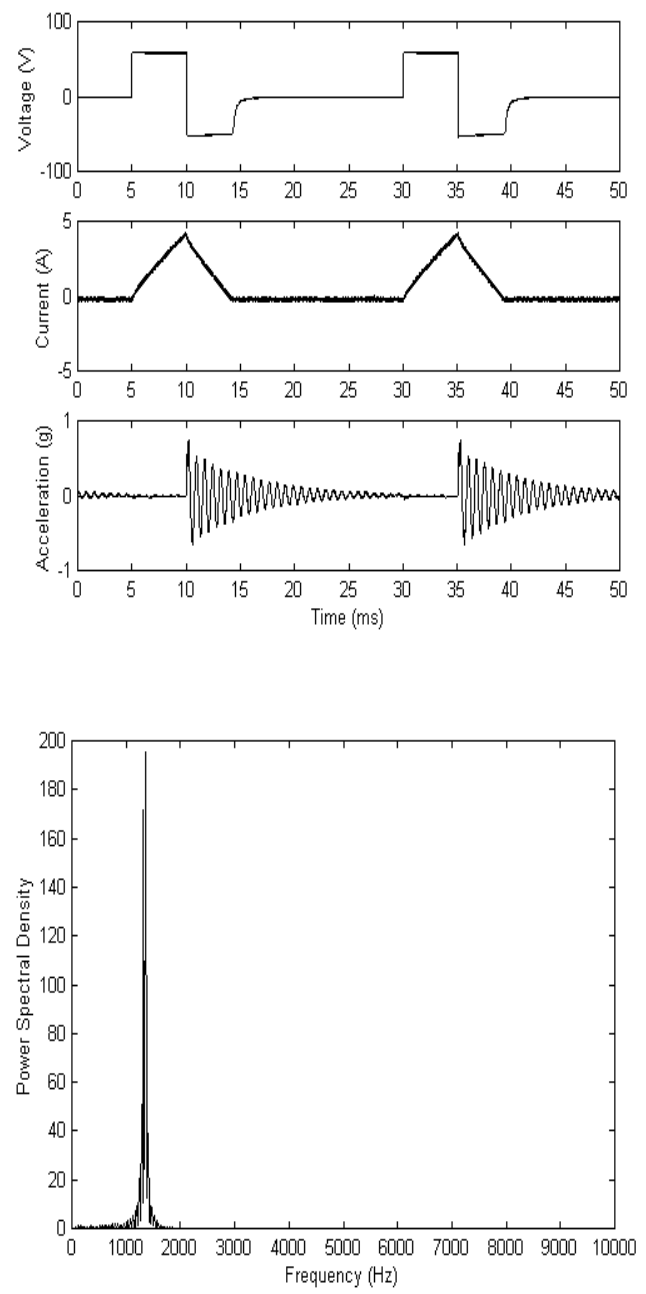

Fig.7 Frequency spectrum under single phase excitation (Repetition=40Hz) Dominant frequency $=1340 \mathrm{~Hz}$

current. The frequency of the exciting current is much lower than the $2^{\text {nd }}$ mode vibration frequency so that the acceleration signals from the accelerometer are allowed to decay to a low enough value during the interval between the adjacent current pulses. Fig.7 shows the waveform of the supply voltage, current pulse and the acceleration in the time domain as well as the power spectrum in the frequency domain. The acceleration waveform clearly demonstrates that the vibrations occur at switch-off of the phase current, instead of during switched-on. This result confirms conclusions drawn in previous papers [2 4].

The calculated results with the analytical formulas and corresponding percentage errors relative to the measured values are given in Table 2 . It should be pointed out that the measured SRM has radiator ribs, which may reduce the resonant frequency of the $2^{\text {nd }}$ order mode shape [1]. The measurement frequency $(1380 \mathrm{~Hz})$ includes the effects of this factor, and almost exactly matches the numerical results 
TABLE 2. COMPARISON TO MEASUREMENT RESULTS

\begin{tabular}{|l|c|c|c|}
\hline Models & Thick Pipe & Thin Ring & Multi-order \\
\hline Frequency $(\mathrm{Hz})$ & 1420 & 1354 & 1405 \\
\hline Error $*(\%)$ & 0.06 & 0.01 & 0.05 \\
\hline
\end{tabular}

<*> The base value is test result $1340 \mathrm{~Hz}$

$(1366 \mathrm{~Hz})$ by finite element model including ribs and terminal box of the machine. The ribs are considered as extra masses attached to the stator yoke in the "multi order model".

\section{CONCLUSION}

The prediction of resonant frequencies is very important for the design of quiet switched reluctance machines and for drive control of low noise SRMs. Two simplified formulas are derived to calculate the $2^{\text {nd }}$ order modal frequency of the SRM, and their applicable ranges are discussed in the paper. A more sophisticated formula can also be used to predict the $0^{\text {th }}, 3^{\text {rd }}$, and $4^{\text {th }}$ order modal frequencies within a certain range of yoke radius/thickness. Through practical measurements and a comparison with numerical results, several useful conclusions are drawn as follows:

The effect of the length of the stator laminations on inplane natural vibration frequencies can be neglected, so that 2D analytical models for a SRM with smooth frame is accurate enough to satisfy the application requirements in the prediction of in-plane vibration modes. The effects of frame ribs have been neglected.

With stator poles and winding space kept unchanged, the $2^{\text {nd }}$ order modal frequency increases linearly with the outer radius of the stator yoke. The multi order model is accurate to a wider range of practical stator dimensions to calculate the modal frequencies. If the ratio of average yoke radius to thickness falls into the 5.4 9.2 range, the thin ring model can be applied to estimate the modal frequency and the error is less than $5 \%$.

The multi order formula can be used to predict the low order modal frequencies. The accuracy of the formulas is high enough for the calculation of the $0^{\text {th }}, 2^{\text {nd }}$ and $4^{\text {th }}$ order (within $R_{y(a v)} / H_{y}=4.5 \sim 7.3$ ) modal frequencies.

The FE results are closer to the experimental results because the complexity of the stator structure must be reduced before developing the analytical models. Additional natural frequencies are found in the numerical results besides those corresponding to the integer order mode shapes.
The measurement results showed that the frame ribs lowered the $2^{\text {nd }}$ order frequency. The measured waveform demonstrated that the $2^{\text {nd }}$ order modal vibration occurred at the turn-off moment of the current pulse, not switched-on, thus validating many former papers [1 4].

Acknowledgements: The authors acknowledge the support of Delphi Research Labs, Shelby Township, Michigan, and the US Navy, through the Office of Naval Research for an equipment research grant.

\section{REFERENCES}

[1] P.Pillay, W. Cai, "An investigation into vibration in switched reluctance motor," Conference Record of the 1998 IEEE Industrial Application Society, $33^{\text {th }}$ IAS Annual Meeting, Vol.1, St. Louis, USA, Oct. 12-16, 1998.

[2] D.E. Cameron, J.H. Lang, S.D.Umans, "The origin and reduction of acoustic noise in doubly salient variable-reluctance motors," IEEE Trans. on Industry Applications, Vol.28, No.6, November/December, 1992, pp.1250 1255.

[3] C. Pollock, C.Y.Wu, "Acoustic noise cancellation techniques for switched reluctance drives," Conference Record of the 1995 IEEE Industrial Application Society, 30 ${ }^{\text {th }}$ IAS Annual Meeting, Vol.1, Orlando, USA, Oct. 8-12, 1995, pp.448-455.

[4] R.S.Colby, F.Mottier, T.J.E.Miller, "Vibration modes and acoustic noise in a 4-phase switched reluctance motor," Conference Record of the 1995 IEEE Industrial Application Society, 30 ${ }^{\text {th }}$ IAS Annual Meeting, Vol.1, Orlando, USA, Oct. 8-12, 1995, pp.441 447.

[5] P.Pillay, R.M.Samudio, M.Ahmed, P.T.Patel, "A Chopper-controlled SRM drive for reduced acoustic noise and improved ride-through capability using super-capacitor," IEEE Trans. on Industry Applications, Vol.31, No.5, September/October, 1995, pp.1029 1038.

[6] Singiresu S. Rao, Mechanical Vibrations ( $3^{\text {rd }}$ edition), ISBN: 0-20152686-7, Addison Wesley, 1995.

[7] Warren C. Young, 'Roark's Formulas for Stress \& Strain (sixth edition)," McGraw-Hill, ISBN 0-07-072541-1, 1989, pp.67, 263.

[8] S.P.Verma, R.S.Girgis, "Method for accurate determination resonant frequencies and vibration behaviour of stators of electrical machines," Proceedings of IEE, Part B, Vol.128, No.1, January 1981, pp.1-11.

[9] Robert D. Blevins, "Formulas for Natural Frequency and Mode Shape," Van Nostrand Reinhold, New York, ISBN 0-442-20710-7, 1979, pp.205.

[10] Shikun Chen, "Electrical Machinery Design ( $1^{\text {st }}$ edition)," Press of Machinery Industry, Beijing, China, 1982 (in Chinese). 